\title{
Collateral Circulation in Spinal Cord Injury: A Comprehensive Review
}

\author{
Ezequiel Garcia-Ballestas ${ }^{1} \quad$ B. V. Murlimanju² $\quad$ Yeider A. Durango-Espinosa ${ }^{1} \quad$ Andrei F. Joaquim ${ }^{3}$ \\ Harold E. Vasquez ${ }^{4}$ Luis Rafael Moscote-Salazar ${ }^{5} \quad$ Amit Agrawal $^{6}$
}

${ }^{1}$ Faculty of Medicine, Center for Biomedical Research (CIB),

University of Cartagena, Cartagena, Colombia

2Department of Anatomy, Kasturba Medical College, Mangalore,

Manipal Academy of Higher Education, Manipal, Karnataka, India

${ }^{3}$ Neurosurgery Division, Cartagena de Indias, Bolivar Department of

Neurology, State University of Campinas, Campinas-Sao Paulo, Brazil

${ }^{4}$ Universidad del Sinu, Cartagena de Indias, Consejo

Latinoamericano de Neurointensivismo (CLaNi),

Cartagena de Indias, Colombia

${ }^{5}$ Neurosurgeon-Critical Care, Center for Biomedical Research (CIB),

Cartagena Neurotrauma Research Group, Faculty of Medicine,

University of Cartagena, Cartagena, Colombia

6 Department of Neurosurgery, All India Institute of Medical

Sciences, Bhopal, Madhya Pradesh, India

\author{
Address for correspondence Luis Rafael Moscote-Salazar, MD, \\ Neurosurgeon-Critical Care, Center for Biomedical Research (CIB), \\ Cartagena Neurotrauma Research Group, Faculty of Medicine, \\ University of Cartagena, Calle de la Universidad, Cra. 6 \#36-100, \\ Cartagena, Bolívar 130001, Columbia \\ (e-mail: rafaelmoscote21@gmail.com).
}

\begin{abstract}
Keywords

- collateral circulation

- reperfusion injury

- spinal cord ischemia

- spinal cord

- spinal injury

Surgery is the most common cause of spinal cord ischemia; it is also caused by hemodynamic changes, which disrupt the blood flow. Direct ligation of the spinal arteries, especially the Adamkiewicz artery is involved as well. Other causes of spinal cord ischemia include arteriography procedures, thoracic surgery, epidural and rachianesthesia, foraminal infiltration, arterial dissection, systemic hypotension, emboligenic heart disease, thoracic disc herniation, and compression. Understanding the vascular anatomy of the spinal cord is essential to develop optimal strategies for preventing ischemic injuries to the spinal cord. During ischemia, a rich network of intra and paraspinal collaterals allow enough blood flow to compensate the intensity of spinal cord ischemia. In case of interruption of flow of a main artery, the collateral artery increases its flow to maintain perfusion to the tissues. Avoiding spinal cord ischemia by using collateral circulation is necessary to prevent the establishment of hypovolemia, hyperthermia and elevations in venous pressures. The objective of this narrative review is to present the current concepts of spinal collateral circulation and its role in the setting of ischemic events, affecting the vascular supply of the spinal cord.
\end{abstract}

\section{Introduction}

Understanding the vascular anatomy of the spinal cord appears to be essential for developing optimal strategies to prevent ischemic injury to the spinal cord. The ischemic injury can be secondary to aorta-related surgeries, spinal procedures, emboligenic heart disease, neoplasms, trauma,

published online

September 29, 2020
DOI https://doi.org/

10.1055/s-0040-1713724

ISSN 0973-0508. and others. ${ }^{1}$ In recent years, few studies have been conducted to understand the role of spinal collateral circulation in ischemic protection of the spinal cord. It was reported that the spinal arteries may develop collateral circulation and this seems to be a determining factor. ${ }^{2}$ During the ischemic process, a rich network of intra and paraspinal collaterals allow enough blood flow to control or decrease the intensity of 
spinal cord ischemia. ${ }^{3,4}$ This may explain the fact that the frequency of infarction of the spinal cord is rare compared with the cerebral infarction, with an incidence of approximately $1 \%$ for the spinal cord ischemia and the brain representing the remaining $99 \% .^{5,6}$ The purpose of this narrative review is to present the current concepts of spinal collateral circulation and its role in the setting of ischemic events, affecting the vascular supply of the spinal cord.

\section{Anatomy of Spinal Collateral Circulation}

The anatomical circulation of the spinal cord was published in 1882 by Albert W. Adamkiewicz, who postulated that arteria radicularis magna is the largest feeder of the anterior spinal artery. ${ }^{1,78}$ The anterior spinal artery is an anastomosis channel in the midline, anterior to the spinal cord. However, this artery is a part of group of 2 to 17 arteries known as segmental arteries. These segmental arteries feed the spinal arterial system by attaching to the spinal arteries (anterior spinal artery or one of the two posterior spinal arteries) along its height. ${ }^{8}$ These arteries are different from radicular arteries that supply the nerve roots. The lumbar artery branches from the abdominal aorta, from which segmental branches will be projected which, in turn, will join the anterior spinal artery to irrigate the lumbar spine and part of the sacral. The Adamkiewicz artery is projected from the posterior intercostal artery, which is a branch of the aorta, and irrigates some of the thoracic segments to the first lumbar segment. With regard to the upper thoracic and cervical spinal cord, the vertebral arteries, deep cervical arteries and ascending cervical arteries (all these branches are from the subclavian artery) provide the segmental branches. It has been shown that the number of segmental arteries that will perfuse a spinal segment is proportional to the cross-sectional area of the gray matter of that segment. ${ }^{7}$ By this logic, thoracic spine is more susceptible to vascular injury than the cervical spine. It is important to note that there are some arteries called epidural arcades, which appear in all spinal cord segments. These are branches of the segmental arteries that cover the dorsal surface of the vertebral body, anastomosing with the rostral and caudal neighboring segment. ${ }^{9}$

\section{Applied Concept of the Collateral Circulation}

Later descriptions of spinal vasculature emphasized on multiple vessels supplying blood from arteries outside the spinal canal. ${ }^{10}$ During the 1970 s, multiple anastomoses from the vascular territories outside the spinal canal, peripheral vessels and even the paraspinal muscles were described. ${ }^{9}$ There are axial vascular networks of perivertebral tissues (paraspinal muscles and others) that anastomose through small vessels to arteries and nourish the spinal cord, such as segmental, subclavian, hypogastric, lumbar, and intercostal arteries and their branches. The collateral connections implies that, in case of interruption of flow of a main artery, the accessory or collateral artery increases its flow to maintain perfusion to the tissues..$^{10,11}$ Among the different vascular organization at different levels of the spinal cord, the level with the least collateral connection is the thoracic one, since it is only irrigated by a single segmental artery and is the narrowest part of the spinal cord..$^{10,12}$ The correlation between the sectional area of the core and the degree of irrigation has been mentioned. ${ }^{7}$ In addition, the anterior spinal cord is more vulnerable to ischemia, since the posterior medulla receives a supply of two posterior arteries with a high-degree of collateral connections. ${ }^{12,13}$ Different studies in animals showed that the interruption of the flow of all segments to the spinal cord did not produce hypoxic intrathecal acidosis or spinal dysfunction, which remained until the subclavian or middle sacral artery clamping was done. ${ }^{11}$

There is a specific case in which the collateral pathway lacks vascular resistance and the main source decreases its flow, which is called steal; for example, in the event of an aortic cross-clamp, drug-induced arteriovenous shunts, or in the case of bleeding from the intercostal artery in an open excluded aortic segment, conditions demonstrated by experimentation. ${ }^{9,14} \mathrm{~A}$ postmortem study in humans demonstrated the permeability of collateral pathways to the spinal cord by injection of dye. ${ }^{14}$ Another study using pig models showed that, after ligation of the segmental arteries, the anterior spinal artery increases its diameter by vasodilation and thus the arterial tree paraspinal vasculature development. This is consistent vascular adaptation in increased quantity and density of arterioles and a change of arrangement, parallel to the spinal cord (collateral maturation) to permit flow from the subclavian arteries rostrally and the hypogastric arteries caudally ${ }^{9,11,15,16}$ Furthermore, pig models recovered quickly due to this collateral vascular supply network. $\cdot{ }^{15}$ Among the 6000 segmental arteries' ligation, there were no cases of paraplegia, so it is inferable that the ischemic event did not take place. ${ }^{10}$ It has been postulated that the collateral spinal system is divided into three compartments: two paraspinal (one of the musculatures itself and another extramuscular) and one intraspinal compartment. The paraspinal compartment turns out to be the largest involved in the long-term collateral circulation. In this compartment, the changes of collateral maturation described above take place once the main supply is cut, which also encloses a reserve mechanism at rest. Once the metabolic demand increases, the vascular adaptation starts increasing the perfusion to the spinal cord. The intraspinal compartment has a collateral mechanism similar to the circle of Willis in the cerebral interpeduncular fossa, so that it is a system, which is involved in short-term collateral circulation and does not have a collateral maturation like paraspinal compartment. Rather, alterations consist in the increase of the caliber of the blood vessels. ${ }^{9,16}$ Epidural arcades in the intraspinal system have an important role in the immediate irrigation function as the most important reserve vessels. ${ }^{16}$

\section{Etiopathogenesis of Spinal Cord Ischemia}

The causes of spinal cord ischemia are numerous. Among them, iatrogenic injuries during the thoracic and thoracoabdominal open aortic aneurysms repair (TOAR) or endovascular aortic repair (TEVAR) are the most common. Other 
etiologies for spinal cord ischemia described are arteriography procedures, thoracic surgery, epidural and rachianesthesia, foraminal infiltration, arterial dissection, systemic hypotension, emboligenic heart disease, thoracic disc herniation, and compressive causes including epidural metastases, meningiomas, trauma and spondyloarthrosis.,5, ${ }^{5,17,18}$ Approximately, $87 \%$ of cases of spinal cord ischemia are related to the compromise of the territory of the anterior spinal artery, ${ }^{5,17}$ and in a few cases, the affected territory is related to the posterior spinal artery. The explanation for this fact is mostly due to the existence of the collateral nature of the posterior circulation spinal system. Spinal cord ischemia can be systemic, which is secondary to systemic hypoperfusion (cardiac arrest or shock), or in a focal area, more common in the territory of the Adamkiewicz artery. ${ }^{5}$

\section{Surgical Procedure}

Surgery is the most common cause of spinal cord ischemia; it is also caused by hemodynamic changes, which disrupt the blood flow. Direct ligation of the spinal arteries, especially the Adamkiewicz artery is involved as well. ${ }^{19}$ In addition, the administration of epidural anesthesia by an unknown mechanism induces volume increase of the administered liquid, adding to the vasoconstrictor effect of the drug and direct traumatic damage, which may lead to ischemia in the spinal cord tissue. ${ }^{20}$

\section{Atherosclerosis, Dissection and Stroke}

The presence of atheromas and emboli in the spinal and radicular arteries is very infrequent. Therefore, atherosclerotic spinal cord infarctions only occur due to severe aortic involvement or dissecting aneurysms. ${ }^{11}$ Cases of spinal cord infarction secondary to arterial dissection have been reported. ${ }^{21}$ Patients with cardioembolic diseases have a greater risk of developing spinal ischemia. There are cases which have been reported in patients with rheumatic mitral valve disease, atrial myxoma, bacterial endocarditis and others. ${ }^{22}$ Fibrocartilaginous emboli have also been reported, and the mechanisms include extrusion of part of the fibrocartilaginous nucleus pulposus material from inside the intervertebral disc to final embolization in one of the spinal cord sinusoids, resulting in spinal cord infarction. ${ }^{23}$

\section{Arteriovenous Malformations}

They consist of persistence of a connection between an artery and a vein without the interposition of a capillary network, which leads to venous congestion, edema and hypertension. This eventually causes chronic venous ischemia with progressive myelopathy or, if the derivation is purely intradural, it can cause hematomyelia and subarachnoid hemorrhage. ${ }^{5}$

\section{Trauma}

Pathophysiological mechanisms in spinal cord ischemia described two notable stages, primary and secondary damage stages. ${ }^{24-26}$ The primary stage is the result of direct mechanical or physical damage to the spinal cord by a combination of compression, laceration, distraction, vascular insult, or shearing. The resulting damage to the microvasculature leads to the production of a progressive edema, ischemia, and activation of proapoptotic signaling. ${ }^{18,27-29}$ The process of secondary damage begins with progressive vascular changes due to the initial trauma. ${ }^{30}$ The endothelial damage prompts the inflammatory cascade of spinal cord ischemia, leading to extravasation of red cells, platelet aggregation and fibrin deposition. These platelet aggregates occlude the vasculature leading to ischemia, and endothelial gaps promote the extravasation of plasma and proteins, thereby causing edema. ${ }^{28,29}$ After the injury, hypoperfusion developed in gray matter of spinal cord extends to the surrounding white matter rostrally and caudally; this hypoperfusion slows or completely blocks the propagation of action potentials along the axons. ${ }^{29}$ Ischemia due to a decrease in perfusion is followed by a phase of hyperemia, which is known as luxury perfusion caused by a reduction in perivascular $\mathrm{pH}$ and is linked to the accumulation of metabolites such as lactate acid. ${ }^{31}$ Finally, the release of superoxide free radicals, nitric oxide destroys cell membranes, consequently leading to cell death, a process known as excitotoxicity. ${ }^{18,25,26,28,29}$

\section{Imaging of Collateral Spinal Circulation}

The identification of collateral circulation in patients with risk factors of developing spinal cord ischemia is important for the surgical planning and prevention of neurological complications. ${ }^{32}$ Among the most commonly used imaging modalities, the multi-detector row computed tomography (MDCT) seems to be the most promising, since it allows the three-dimensional reconstruction of images of the organ studied. In a recent study, collateral circulation could be identified by MDCT in $83 \%$ of patients with segmental artery occlusion in the artery originating from the artery of Adamkiewicz. ${ }^{32}$ It was also reported that the use of other modalities, such as CT angiography (CTA) and magnetic resonance angiography (MRA), in order to check the collateral circulation to the Adamkiewicz artery through the intercostal artery, thoracodorsal artery, internal thoracic artery and inferior phrenic artery in patients with extensive degenerative thoracoabdominal aortic aneurysm, ${ }^{32}$ reported detection rates between 67 to $93 \%{ }^{7,33}$ However, the effectiveness is limited to the collateral paths exceeding the millimeter in diameter, and these also lack functional validation. ${ }^{9}$ However MRA is recommended for patients in whom the MDCT could not identify the Adamkiewicz artery. ${ }^{32}$

\section{Somatosensory-evoked Potentials (SEPs) and Motor}

The belief among spine surgeons is that monitoring the spinal cord function during the procedure reduces the likelihood of injuring the spinal cord iatrogenically. However, there is no recognition to support its use as a standardized procedure, although there are centers which consider it as a part of 
routine. While it is true that some of the ischemic changes are delayed to be detected during operation, those somatosensory evoked potentials (SEPs) that cannot be seen during this time can be an important tool during the patient unconsciousness postoperatively..$^{10,14,34}$ SEPs are defined as brain electrical response to a somatosensory stimulus in order to detect neural integrity of ascending neural pathways; this technique, which was used for 40 years, provides preliminary warnings about the changes in spinal cord function..$^{10,35}$ Due to the transient changes in the perfusion of spinal cord during the surgery, it is possible that changes are registered in the SEP. But after 15 minutes of the perfusion, function recovers without resulting in paraplegia. ${ }^{10,14}$ The disadvantage of this technique is that when evaluating only ascending tracts (sensory), ischemic injury of descending tracts (motor) is not detectable, so motor-evoked potentials (MEPs) play a key role in interpreting these studies. . $^{10,13,14,35}$ Its advantages with respect to SEP are superior specificity and sensitivity for detecting the ischemia in risk surgeries, and its disadvantages are high cost and convenience. ${ }^{10,14}$ Furthermore, with the spinal cord ischemia being more common in the anterior portion for the reasons mentioned above, MEPs are most relevant to SEPs. ${ }^{13}$ The recommendation is to simultaneously use both MEP and SEP to increase the diagnostic capacity of these studies. ${ }^{35}$

\section{Infrared Spectroscopy}

Due to the invasive nature of the evoked potentials which were described above, an alternative method for measuring the noninvasive spinal perfusion is practiced, indirect infrared spectroscopy. ${ }^{4}$ It is a tool that reflects the nature of the collateral circulation of the spinal cord, measuring the degree of oxygenation of the paraspinal muscles and providing a rough estimate of the oxygenation of the spinal cord. Any change detected by this system can be an ischemic injury to the spinal cord. ${ }^{11,36}$ Research is performed to check the perfusion relationship between the paraspinal muscles and spinal cord to validate the indirect nature of this study. ${ }^{4}$

\section{Collateral Spinal Circulation and Therapeutic Strategies}

To avoid spinal cord ischemia using the collateral circulation, it is necessary to prevent the establishment of hypovolemia, hyperthermia, elevations in venous pressures, and other threats that constitute risk factors. ${ }^{8,37}$ One of the major concerns during the thoracoabdominal surgery is spinal cord injury with the sacrifice of the segmental arteries, which play a crucial role in the blood supply of spinal cord. ${ }^{3}$ Due to this, many alternatives have been proposed to reduce the mortality and morbidity associated with spinal cord injury, including the neurological complications such as paraplegia and paraparesis. ${ }^{11}$ These methods include pharmacological procedures, distal perfusion, hypothermia, reimplantation of segmental arteries, and cerebrospinal fluid (CSF) drainage. ${ }^{38}$

\section{Maintenance of Mean Arterial Pressure}

Whenever there is decreased blood flow by any etiologic factor, collateral circulation plays a crucial role and one of the means for strengthening mechanisms involves maintaining the mean arterial pressure over the physiological upper limit. ${ }^{5,12,14,15,36,39}$ Catapano et $\mathrm{al}^{39}$ analyzed that there is a positive association between high values of mean arterial pressure and neurological improvement in patients with spinal cord injury. Ullery et al ${ }^{40}$ came to the same conclusion in patients who had undergone a correction of aortic aneurysm; they also added that the mean arterial pressure $<70 \mathrm{~mm} \mathrm{Hg}$ is a risk factor for spinal cord ischemia. The goal is to keep the mean arterial pressure at least $80 \mathrm{~mm} \mathrm{Hg}$ for a surgical procedure that produces spinal ischemia, such as an aorta-related procedure, and maintain this goal by suspending 24 to 72 hours, adding any hypertensive agent if necessary. In the event of neurological symptoms, target changes to be aggressively incorporated to at least $100 \mathrm{~mm} \mathrm{Hg}$, and antihypertensive treatment will be retaken up over a period of 1 to 3 months. ${ }^{37}$ In general, this measure is well adopted, although clearly recognizing the fact that the etiological factor determines the effectiveness. This will have to be done to avoid the damage perpetuated by ischemic processes because different pathological processes can lead to a spinal cord infarction.,33 The transfusion is indicated to stay hemoglobin values higher at 10 to $12 \mathrm{mg} / \mathrm{dL}$ in the early postoperative period with the appropriate coagulation profile. ${ }^{37}$

\section{Hypothermia}

Temperature plays a crucial role in the metabolism of oxygen. It should be noted that oxygen participates in the reactions of cell respiration and cell death, so that interruption of use is key to prevent the neuronal damage after the blood supply is suspended. ${ }^{40}$ Hypothermia is neuroprotective as it seeks to reduce the rate of oxygen metabolism. ${ }^{40}$ Hypothermia reduces intracellular enzyme activity by 6 to $7 \%$ per degree of temperature reduction; also, it reduces glutamate release or enhanced release of GABA, suppresses intracellular calcium intake, and decreases free radical generation to result in mitigation of harmful response of reperfusion. ${ }^{37,40}$ There is little evidence to support this practice in endovascular procedures, but abundant in open surgical repair of aortic aneurysms. ${ }^{37}$ By the foregoing, the intraoperative use of cardiopulmonary bypass (feasible through the femoral artery, or according to the current trend, of the right axillary artery) in moderate temperature (taken in the nasopharynx of $20-28^{\circ} \mathrm{C}$ ) is a measure that requires vast technic and the monitoring of certain physiological variables such as coagulation, acid-base balance and glycemia. ${ }^{40}$

\section{Cerebrospinal Fluid Drainage}

The drainage of cerebrospinal fluid (CSF) by means of the posture of a lumbar cannula of the intrathecal space produces improvement of the neurological outcomes when it is a procedure related to the aorta. ${ }^{12,36}$ Spinal perfusion is a 
function of mean arterial pressure minus the pressure of the CSF. When the CSF pressure exceeds the spinal arterial pressure, a critical closing pressure is achieved and the veins collapse regardless of the inlet pressure, ${ }^{34}$ thus the flow to bone is reduced because the arteries are unable to pass the blood veins. In this order of ideas, by reducing intrathecal pressure to balance this equation, we aim to increase spinal perfusion to reduce the risk of ischemia. ${ }^{5,34}$ It does not constitute an isolated measure; rather, it requires the concomitance of other preventive measure of ischemia, since studies indicate that this way is effective. ${ }^{34}$

\section{Neuroprotective Drugs}

The use of these drugs is aimed at reducing overall spinal cord damage, achieving their goal by reducing metabolic needs, restricting neuronal apoptosis, mitigating the inflammatory process, and increasing the tolerance of spinal ischemia. ${ }^{37}$ It is important to note that there is still little evidence of benefit for clinical application in humans, although studies in experimental results are incriminating. Glucocorticoids are commonly used due to their anti-inflammatory capacity; they manage to reduce edema and chemotaxis of polymorphonuclear site of injury. ${ }^{41}$ However, a Cochrane review found insufficient evidence for the use of steroids as neuroprotective in ischemic strokes. ${ }^{42}$ Papaverine given perispinally increases the blood flow through its vasodilatory capacity, thereby decreasing the ischemic injury. The use of this medication reduced the spinal cord injury mechanism described above after repair of TOAR. ${ }^{37}$ In some animal studies, propofol has been shown to be neuroprotective, perhaps because of its antiapoptotic, antioxidant or anti-inflammatory nature. ${ }^{43}$ The use of lidocaine as neuroprotective is controversial, despite their ability to reduce neuronal metabolism, improve cerebral blood flow and possess anti-inflammatory effects. ${ }^{40,43}$ Another drug with potential neuroprotective effects is mannitol, which has an osmotic, antiapoptotic and free radical scavenging effect. ${ }^{40}$ The benefits of this drug was studied, but there is inconclusive data about it and its effects in the patient group..$^{44}$

\section{Conclusions}

The spinal cord circulation is poorly studied in comparison to the blood supply of brain. The etiopathological factors of spinal cord ischemia are numerous. Iatrogenic injuries during TOAR and TEVAR are the most common. Understanding the blood supply of the spinal cord and medical interventions, which may prevent ischemia, is of paramount importance to avoid iatrogenic spinal cord injuries. Clinical and experimental studies are necessary to improve the knowledge of this challenging subject.

\section{Conflict of Interest}

None declared.

\section{References}

1 Etz CD, Kari FA, Mueller CS, et al. The collateral network concept: a reassessment of the anatomy of spinal cord perfusion. J Thorac Cardiovasc Surg 2011;141(4):1020-1028

2 Salkov M, Tsymbaliuk V, Dzyak L, Rodinsky A, Cherednichenko Y, Titov G. New concept of pathogenesis of impaired circulation in traumatic cervical spinal cord injury and its impact on disease severity: case series of four patients. Eur Spine J 2016;25(Suppl 1):11-18

3 Kise Y, Kuniyoshi Y, Inafuku H, Nagano T, Hirayasu T, Yamashiro S. Directly measuring spinal cord blood flow and spinal cord perfusion pressure via the collateral network: correlations with changes in systemic blood pressure. J Thorac Cardiovasc Surg 2015;149(1):360-366

4 Luehr M, Mohr FW, Etz CD. Indirect neuromonitoring of the spinal cord by near-infrared spectroscopy of the paraspinous thoracic and lumbar muscles in aortic surgery. Thorac Cardiovasc Surg 2016;64(4):333-335

5 Saliou G, Krings T. Vascular diseases of the spine. Handb Clin Neurol 2016;136:707-716

6 Yasui H, Ozawa N, Mikami S, et al. Spinal cord ischemia secondary to epidural metastasis from small cell lung carcinoma. Am J Case Rep 2017;18:276-280

7 Bosmia AN, Tubbs RS, Hogan E, et al. Blood supply to the human spinal cord: part II. Imaging and pathology. Clin Anat 2015;28(1):65-74

8 Griepp EB, Di Luozzo G, Schray D, Stefanovic A, Geisbüsch S, Griepp RB. The anatomy of the spinal cord collateral circulation. Ann Cardiothorac Surg 2012;1(3):350-357

9 Meffert P, Bischoff MS, Brenner R, Siepe M, Beyersdorf F, Kari FA. Significance and function of different spinal collateral compartments following thoracic aortic surgery: immediate versus long-term flow compensation. Eur J Cardiothorac Surg 2014;45(5):799-804

10 Wu L, Qiu Y, Ling W, Shen Q. Change pattern of somatosensory-evoked potentials after occlusion of segmental vessels: possible indicator for spinal cord ischemia. Eur Spine J 2006;15(3):335-340

11 Dias-Neto M, Reis PV, Rolim D, Ramos JF, Teixeira JF, Sampaio S. Strategies to prevent TEVAR-related spinal cord ischemia. Vascular 2017;25(3):307-315

12 Colman MW, Hornicek FJ, Schwab JH. Spinal cord blood supply and its surgical implications. J Am Acad Orthop Surg 2015;23(10):581-591

13 Shine TS, Harrison BA, De Ruyter ML, et al. Motor and somatosensory evoked potentials: their role in predicting spina cord ischemia in patients undergoing thoracoabdominal aortic aneurysm repair with regional lumbar epidural cooling. Anesthesiology 2008;108(4):580-587

14 Griepp RB, Griepp EB. Spinal cord perfusion and protection during descending thoracic and thoracoabdominal aortic surgery: the collateral network concept. Ann Thorac Surg 2007;83(2):S865-S869, discussion S890-S892

15 Griepp EB, Griepp RB. The collateral network concept: minimizing paraplegia secondary to thoracoabdominal aortic aneurysm resection. Tex Heart Inst J 2010;37(6):672-674

16 Kari FA, Wittmann K, Saravi B, et al. Immediate spinal cord collateral blood flow during thoracic aortic procedures: the role of epidural arcades. Semin Thorac Cardiovasc Surg 2016;28(2):378-387

17 Peckham ME, Hutchins TA. Imaging of vascular disorders of the spine. Radiol Clin North Am 2019;57(2):307-318

18 Winter B, Pattani H. Spinal cord injury. Anaesth Intensive Care Med 2011;12:403-405 
19 Berg P, Kaufmann D, van Marrewijk CJ, Buth J. Spinal cord ischaemia after stent-graft treatment for infra-renal abdominal aortic aneurysms. Analysis of the Eurostar database. Eur J Vasc Endovasc Surg 2001;22(4):342-347

20 Chan LL, Kumar AJ, Leeds NE, Forman AD. Post-epidural analgesia spinal cord infarction: MRI correlation. Acta Neurol Scand 2002;105(4):344-348

21 Wu Y, Li W, Xie X, Jing Z, Lu W, Huang L. Endovascular treatment with tirofiban during the acute stage of cervical spinal cord infarction due to vertebral artery dissection. J Spinal Cord Med 2020;43(1):130-133

22 Rigney L, Cappelen-Smith C, Sebire D, Beran RG, Cordato D. Nontraumatic spinal cord ischaemic syndrome. J Clin Neurosci 2015;22(10):1544-1549

23 AbdelRazek M, Elsadek R, Elsadek L. Case series of two patients with Fibrocartilaginous Embolism mimicking Transverse Myelitis of the Spinal Cord. J Clin Neurosci 2017;40:66-68

24 Ahuja CS, Martin AR, Fehlings M. Recent advances in managing a spinal cord injury secondary to trauma. F1000 Res 2016;5:5

25 Ahuja CS, Nori S, Tetreault L, et al. Traumatic spinal cord injury-Repair and regeneration. Neurosurgery 2017;80(3S): S9-S22

26 Rogers WK, Todd M. Acute spinal cord injury. Best Pract Res Clin Anaesthesiol 2016;30(1):27-39

27 Rouanet C, Reges D, Rocha E, Gagliardi V, Silva GS. Traumatic spinal cord injury: current concepts and treatment update. Arq Neuropsiquiatr 2017;75(6):387-393

28 Ballesteros Plaza V, Marré Pacheco B, Martínez Aguilar C, Fleiderman Valenzuela J, Zamorano Pérez JJ. Lesión de la médula espinal: actualización bibliográfica: fisiopatología y tratamiento inicial. Coluna/Columna 2012;11:73-76

29 Mataliotakis GI, Tsirikos AI. Spinal cord trauma: pathophysiology, classification of spinal cord injury syndromes, treatment principles and controversies. Orthop Trauma 2016;30:440-449

30 Kim Y-H, Ha K-Y, Kim S-I. Spinal cord injury and related clinical trials. Clin Orthop Surg 2017;9(1):1-9

31 Lee J, Thumbikat P. Pathophysiology, presentation and management of spinal cord injury. Surgery 2015;33:238-247

32 Domoto S, Kimura F, Asakura T, Nakazawa K, Koike H, Niinami H. Intraspinal collateral circulation to the artery of Adamkiewicz detected with intra-arterial injected computed tomographic angiography. J Vasc Surg 2016;63(6):1631-1634
33 Bosmia AN, Hogan E, Loukas M. Tubbs RS, Cohen-Gadol AA. Blood supply to the human spinal cord: part I. Anatomy and hemodynamics. Clin Anat 2015;28(1):52-64

34 Godet G, Bertrand M, Fléron MH, et al. Cerebrospinal fluid drainage and thoracic endovascular aneurysm repair. Asian Cardiovasc Thorac Ann 2017;25(9):608-617

35 Ji Y, Meng B, Yuan C, Yang H, Zou J. Monitoring somatosensory evoked potentials in spinal cord ischemia-reperfusion injury. Neural Regen Res 2013;8(33):3087-3094

36 von Aspern K, Haunschild J, Hoyer A, et al. Non-invasive spinal cord oxygenation monitoring: validating collateral network near-infrared spectroscopy for thoracoabdominal aortic aneurysm repair. Eur J Cardiothorac Surg 2016;50(4):675-683

37 Miranda V, Sousa J, Mansilha A. Spinal cord injury in endovascular thoracoabdominal aortic aneurysm repair: prevalence, risk factors and preventive strategies. Int Angiol 2018;37(2): 112-126

38 DeAnda A Jr, Roughneen PT. Spinal cord collateral pathways-The road not taken. Semin Thorac Cardiovasc Surg 2016; 28(2):388-389

39 Catapano JS, John Hawryluk GW, Whetstone W, et al. Higher mean arterial pressure values correlate with neurologic improvement in patients with initially complete spinal cord injuries. World Neurosurg 2016;96:72-79

40 Fernández Suárez FE, Fernández Del Valle D, González Alvarez A, Pérez-Lozano B. Intraoperative care for aortic surgery using circulatory arrest. J Thorac Dis 2017;9(Suppl 6) :S508-S520

41 Wu GJ, Chen WF, Sung CS, et al. Preventive effects of intrathecal methylprednisolone administration on spinal cord ischemia in rats: the role of excitatory amino acid metabolizing systems. Neuroscience 2007;147(2):294-303

42 Keenan JE, Benrashid E, Kale E, Nicoara A, Husain AM, Hughes GC. Neurophysiological intraoperative monitoring during aortic arch surgery. Semin Cardiothorac Vasc Anesth 2016;20(4): 273-282

43 Manetta F, Mullan CW, Catalano MA. Neuroprotective strategies in repair and replacement of the aortic arch. Int J Angiol 2018;27:98-109

44 Krüger T, Hoffmann I, Blettner M, Borger MA, Schlensak C, Weigang E; GERAADA Investigators. Intraoperative neuroprotective drugs without beneficial effects? Results of the German Registry for Acute Aortic Dissection Type A (GERAADA) Eur J Cardiothorac Surg 2013;44(5):939-946 